\title{
Dosimetric Analysis of Three Different Radiotherapy Techniques in Patients with Breast Cancer and Their Impact on Organs at Risk (OAR)
}

\author{
Vikas Jagtap ${ }^{1 *}$, Dimpal Saikia ${ }^{2}$, Shashi Bhushan Sharma ${ }^{3}$, Shayori Bhattacharjee ${ }^{4}$, \\ Moirangthem Nara Singh ${ }^{3}$, Sachindra Goswami ${ }^{3}$, Apurba Kumar Kalita ${ }^{3}$
}

\author{
${ }^{1}$ Department of Radiation Oncology, North Eastern Indira Gandhi Regional Institute of Health and Medical Sciences, Shillong, \\ India \\ ${ }^{2}$ Department of Radiation Oncology, Cancer Hospital, Gauhati Medical College, Guwahati, India \\ ${ }^{3}$ Department of Radiation Oncology \& Medical Physics, Dr. B Borooah Cancer Institute, Guwahati, India \\ ${ }^{4}$ Department of Radiation Oncology, American Oncology Institute, Nagpur, India \\ Email: *drvikasj@yahoo.co.in
}

How to cite this paper: Jagtap, V., Saikia, D., Sharma, S.B., Bhattacharjee, S., Singh, M.N., Goswami, S. and Kalita, A.K. (2018) Dosimetric Analysis of Three Different Radiotherapy Techniques in Patients with Breast Cancer and Their Impact on Organs at Risk (OAR). International Journal of Medical Physics, Clinical Engineering and Radiation Oncology, 7, 131-140. https://doi.org/10.4236/ijmpcero.2018.72011

Received: February 1, 2018

Accepted: March 12, 2018

Published: March 15, 2018

Copyright @ 2018 by authors and Scientific Research Publishing Inc. This work is licensed under the Creative Commons Attribution International License (CC BY 4.0).

http://creativecommons.org/licenses/by/4.0/ (c) (i) Open Access

\begin{abstract}
Aim: Dosimetric analysis of three different Radiotherapy techniques in patients with Breast Cancer and their impact on OAR's. Materials and Methods: 12 patients of Carcinoma Breast who received breast radiotherapy were selected for analysis. Computed tomography (CT) simulation image data sets were retrieved. Planning target Volume (PTV), heart and ipsilateral lung were contoured for planning and analysis of doses. Three different plans using conventional bi-tangential fields were prepared, Plan 1 with SAD full beam with wedge (SAD-FBW), Plan 2 with SSD half beam with wedge (SSD-HBW) and plan 3 with SSD half beam without wedge techniques (SSD-HBO) in CMS Xio TPS. Statistical analysis was done using SPSS version 16.0. Results: The PTV coverage was significantly better in SAD-FBW techniques when compared with the other two techniques i.e. SSD-HBW (mean $=92.33, \mathrm{SD}=4.69$, $\mathrm{p}=0.005$ ) and SSD-HBO (mean $=75.05, \mathrm{SD}=11.92, \mathrm{p}=0.002$ ). The mean heart doses were significantly better in SAD-FBW compared to SSD-HBW (mean $=3.75, \mathrm{SD}=2.27, \mathrm{p}=0.017$ ) but in SSD-HBO technique mean heart doses were better than SAD-FBW technique (mean $=3.27, \mathrm{SD}=1.94, \mathrm{p}=0.004$ ). Similarly, the left lung V20 values were significantly better in SSD-HBO technique than $\mathrm{SAD}-\mathrm{FBW}$ technique (mean $=11.75, \mathrm{SD}=4.34, \mathrm{p}=0.004$ ) but there was statistically insignificant difference between the SAD-FBW and SSD-HBW techniques. The treatment monitor units were significantly less in SAD-FBW compared to SSD-HBW but SSD-HBO has less MU compared to SAD-FBW
\end{abstract}


technique. Conclusion: SAD Full Beam with wedge technique is practically better than SSD Half Beam technique with or without wedge without compromising PTV coverage considering other dosimetric parameters. We also recommend that centers treating patient with half beam SSD techniques should shift immediately to SAD techniques.

\section{Keywords}

Breast Radiotherapy, Dosimetric Analysis, Breast Cancer

\section{Introduction}

Breast cancer is the most common female cancer in the India with an ASR (Age Standardized Rate) of 25.8 as per Globocan 2012 [1]. While the age adjusted incidence rate of breast cancer in India is lower than the western countries, because of the large population burden of breast cancer is high. Trials comparing breast conserving surgery alone to breast-conserving surgery plus radiation confirmed a substantial decrease in the risk of local recurrence and the prevention of mastectomy with breast irradiation [2]. The most recent analysis of Early Breast Cancer Trialists Collaborative Group (EBCTCG) included individual patient data from 45,000 women in 86 randomized trials. Provisional results from this overview show that radiotherapy after breast surgery reduced the 5 year local recurrence by $15.7 \%$ and the 15 year breast cancer mortality by $4.2 \%$ and that post mastectomy radiotherapy for node-positive disease reduced the 5 year local recurrence by $19.3 \%$ and the 20 year breast cancer mortality by $6.3 \%$ [3] [4].

One of the aims in modern radiotherapy is to avoid early and late side effects associated with treatment. This can be achieved by steep dose gradients between the target volume and the surrounding normal tissues. Adjuvant conventional radiotherapy after breast conserving surgery is based on a tangential technique with two photon beams targeting the residual breast. Wedge filters modulating radiation fluence across the beams are commonly used to compensate for dose inhomogeneity due to irregular target shape [5]. Conventional Tangential Field or Field-in-Field techniques are generally employed to treat breast cancer patients. IMRT techniques with multiple beams increase the low dose volume in lung as well as contra lateral breast. The main concern of IMRT is increase in healthy soft tissue dose which might cause an increased risk of late secondary malignancy [6].

In India many centers still use the conventional tangential beam techniques with breast cone after clinical field marking. The breast cone aids in delivering the treatment by half beam block without the use of wedges. Some centers having Linear accelerators or compatible cobalt machines are also using half beam block technique with use of asymmetric jaw with or without wedge.

Our aim of this study is to do a comparative analysis of three different conventional radiotherapy techniques of breast radiotherapy, i.e. SAD full beam with 
wedge technique (SAD-FBW), SSD half beam with wedge technique (SSD-HBW) and SSD half beam open technique (SSD-HBO).

\section{Materials and Methods}

In this retrospective study twelve patients of left sided breast cancer who have undergone Breast Conservation Surgery with axillary dissection or MRM (Modified Radical Mastectomy) and were given post-operative radiotherapy between October 2016 and February 2017 were selected for analysis. The study was conducted at Dr. B Borooah Cancer Institute. The planning CT scan images of the patients were retrieved and three different types of planning were done. As per our institutional standard protocol the planning CT scan images were taken in supine position with breast board and the hand above the head (Figure 1). For the easier localization of the breast tissue radio-opaque metal wires were used to define the breast contour or field edge. For patients planned for chest wall radiotherapy the metal wires were kept at the clinical skin marking, i.e. superior, inferior, medial and lateral as per the treating clinician's direction. Planning CT scan with 3 - $5 \mathrm{~mm}$ slice thickness was done from the level of mandible up to mid abdomen or $5 \mathrm{~cm}$ below the inferior field border as per skin marking [7] [8]. The CT data were transferred to the CMS Xio 4.80 treatments planning system and then contouring were done by Radiation Oncologists. PTV (Planning Target Volume for chest wall or Breast and OAR's (Organs at Risk) like heart and ipsilateral lung were contoured. Planning Target Volumes (PTV) and Heart was contoured as per the guideline described by RTOG breast cancer atlas [9] and Mary Feng et al. [10] respectively. For lung auto segmentation tool of TPS was used to contour the outline of ipsilateral lung. Plans were done using conventional

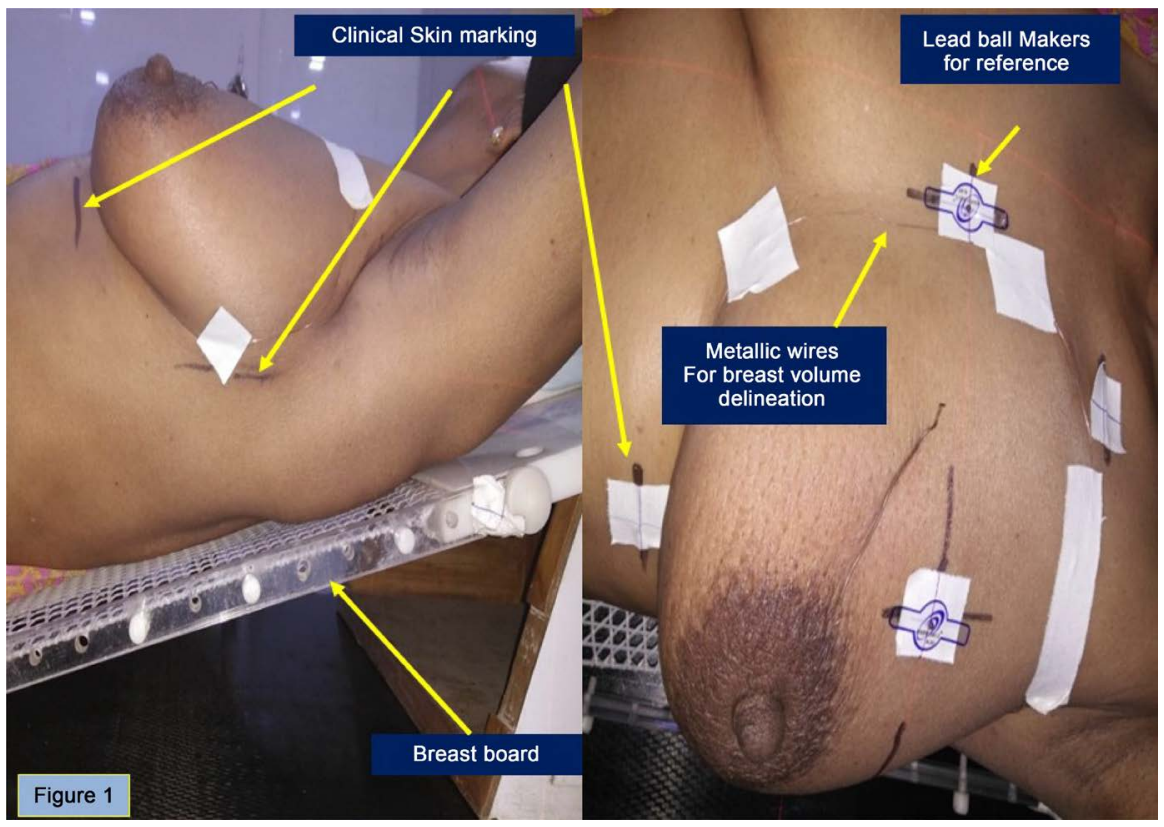

Figure 1. Breast simulation with appropriate skin marking and metallic wires to delineate target volume. 
bi-tangential fields with $6 \mathrm{MV}$ photons. All patients were planned with $40 \mathrm{~Gy}$ in 15 fractions. As breast/ chest wall tissue is a curved volume over chest, tangential fields are suitable to attain a good dose distribution and with the use of tangential fields non-thoracic structures are avoided as much as possible [11].

For SAD-FBW technique, the dose was prescribed at isocenter [12] [13] [14]. Optimum wedge angles, collimator angles, with Clarkson dose calculation algorithm were used during planning to achieve optimal target coverage with minimal hot spot. For SSD-HBW and SSD-HBO technique, almost all the parameters were same except the application of wedge in the former one. In both SSD techniques, beam prescription point was located at $1 / 3$ of the depth from the midpoint of the central axis of the beam to the maximum convexity of the breast/chest wall surface (Figure 2). Dose volume parameters and other parameters were noted from DVH (Dose Volume Histogram). Statistical analysis was done using SPSS version 16.0.

\section{Results}

The various DVH parameters, OAR doses and treatment monitor unit values obtained in 3 different plans are shown in Table 1. The comparison of PTV coverage for the 3 plans is shown in Table 2 . Data were statistically analyzed using Wilcox Signed-Rank test. For analysis SAD-FBW technique was compared with the other two techniques. The PTV coverage was significantly better in SAD-FBW techniques when compared with the other two techniques i.e. SSD-HBW and SSD-HBO. The mean heart doses were significantly better in SAD-FBW compared to SSD-HBW but in SSD-HBO technique mean heart doses were better than SAD-FBW technique but with unacceptable PTV coverage. Left lung V20 values were significantly better in SSD-HBO technique than SAD-FBW technique but there was statistically insignificant difference between the SAD-FBW and SSD-HBW techniques. The treatment monitor units were significantly better

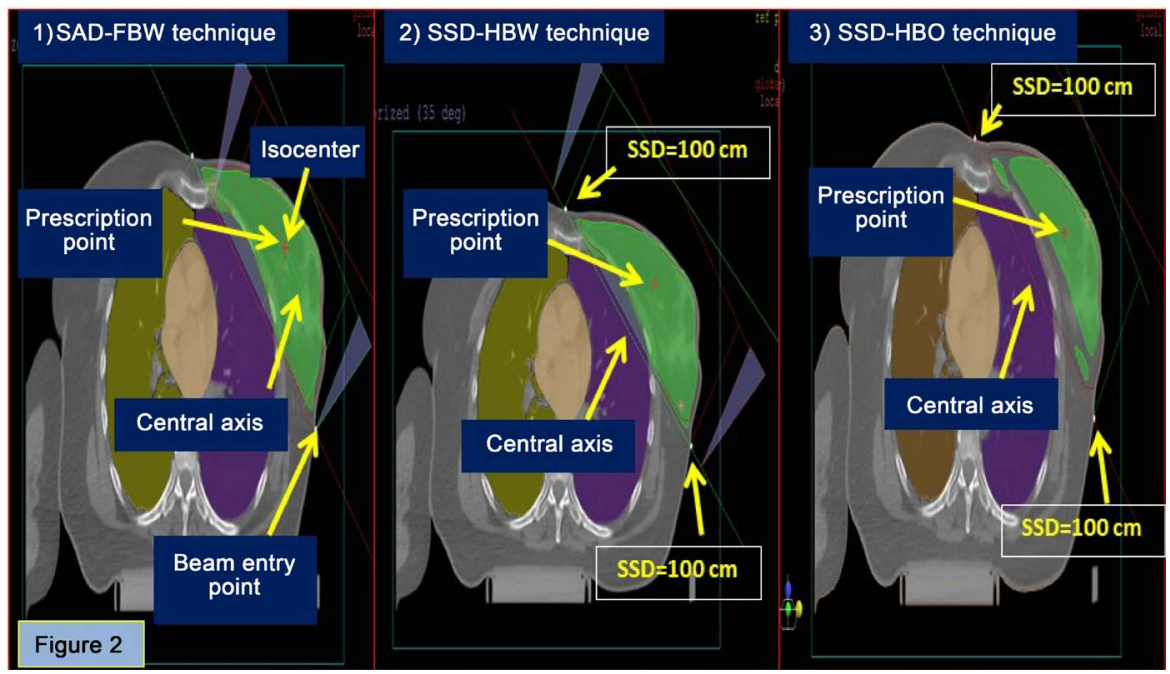

Figure 2. Bi-tangential plans for 3 different techniques 1) SAD-FBW technique, 2) SSD-HBW technique, 3) SSD-HBO technique. 
Table 1. Various parameters showing values for 3 different plans.

\begin{tabular}{|c|c|c|c|c|c|c|c|}
\hline $\begin{array}{l}\text { Patient's } \\
\text { Sl. No. }\end{array}$ & Technique & $\begin{array}{c}\text { PTV } \\
\text { COVERAGE (\%) }\end{array}$ & $\begin{array}{l}\text { V107 } \\
\text { (cc) }\end{array}$ & $\begin{array}{l}\text { V110 } \\
\text { (cc) }\end{array}$ & MU & $\begin{array}{l}\text { Left Lung } \\
\text { V20 (cc) }\end{array}$ & $\begin{array}{c}\text { Mean } \\
\text { Heart Dose (Gy) }\end{array}$ \\
\hline \multirow[t]{3}{*}{1} & SAD-FBW & 92.35 & 29.44 & 5.47 & 662.8 & 131.86 & 4.2 \\
\hline & SSD-HBW & 89.07 & 14.62 & 0.00 & 1040.7 & 133.34 & 4.32 \\
\hline & SSD-HBO & 73.15 & 16.86 & 0.74 & 336.5 & 128.03 & 3.83 \\
\hline \multirow[t]{3}{*}{2} & SAD-FBW & 92.1 & 15.29 & 5.32 & 1068.9 & 83.54 & 3.71 \\
\hline & SSD-HBW & 81.83 & 9.21 & 1.22 & 1528.8 & 86.02 & 3.96 \\
\hline & SSD-HBO & 58.73 & 6.63 & 0.00 & 337.7 & 79.89 & 3.35 \\
\hline \multirow[t]{3}{*}{3} & SAD-FBW & 95.54 & 154.49 & 35.59 & 358 & 41.49 & 2.41 \\
\hline & SSD-HBW & 94.42 & 73.70 & 14.85 & 743 & 41.18 & 2.59 \\
\hline & SSD-HBO & 60.42 & 18.78 & 0.33 & 325 & 37.47 & 2.18 \\
\hline \multirow[t]{3}{*}{4} & SAD-FBW & 96.62 & 465.73 & 195.73 & 289 & 169.16 & 4.69 \\
\hline & SSD-HBW & 96.6 & 286.23 & 79.67 & 614 & 171.16 & 5.1 \\
\hline & SSD-HBO & 86.31 & 192.56 & 71.46 & 343 & 165.24 & 4.56 \\
\hline \multirow[t]{3}{*}{5} & SAD-FBW & 96.47 & 130.91 & 10.23 & 459.8 & 107.04 & 0.66 \\
\hline & SSD-HBW & 96.97 & 70.52 & 4.26 & 948.7 & 111.54 & 0.96 \\
\hline & SSD-HBO & 68.73 & 48.54 & 7.70 & 347.9 & 102.78 & 0.75 \\
\hline \multirow[t]{3}{*}{6} & SAD-FBW & 96.38 & 36.10 & 3.04 & 542 & 162.23 & 1.52 \\
\hline & SSD-HBW & 95.73 & 42.46 & 3.87 & 752.4 & 160.66 & 1.6 \\
\hline & SSD-HBO & 93.56 & 53.19 & 15.07 & 347.8 & 157.60 & 1.49 \\
\hline \multirow[t]{3}{*}{7} & SAD-FBW & 92.4 & 9.50 & 0.00 & 1067.8 & 94.16 & 5.5 \\
\hline & SSD-HBW & 87.04 & 4.12 & 0.00 & 1552 & 99.20 & 5.9 \\
\hline & SSD-HBO & 85.15 & 41.08 & 18.56 & 360.2 & 94.16 & 5.34 \\
\hline \multirow[t]{3}{*}{8} & SAD-FBW & 93.37 & 8.86 & 0.37 & 1069.5 & 54.47 & 3.9 \\
\hline & SSD-HBW & 89.76 & 0.00 & 0.00 & 1303.8 & 53.94 & 3.82 \\
\hline & SSD-HBO & 67.81 & 1.25 & 0.00 & 332 & 50.45 & 3.39 \\
\hline \multirow[t]{3}{*}{9} & SAD-FBW & 96.16 & 32.08 & 0.02 & 598.4 & 157.00 & 0.74 \\
\hline & SSD-HBW & 95 & 33.51 & 1.23 & 1048.5 & 155.92 & 0.65 \\
\hline & SSD-HBO & 89.08 & 19.98 & 3.48 & 344.6 & 150.45 & 0.58 \\
\hline \multirow[t]{3}{*}{10} & SAD-FBW & 98.78 & 8.11 & 0.00 & 1119.5 & 56.48 & 3.81 \\
\hline & SSD-HBW & 95.94 & 0.00 & 0.00 & 1563.3 & 54.72 & 3.71 \\
\hline & SSD-HBO & 75.21 & 19.00 & 3.50 & 356.5 & 50.54 & 3.22 \\
\hline \multirow[t]{3}{*}{11} & SAD-FBW & 95.44 & 42.42 & 20.04 & 920.5 & 131.41 & 8.7 \\
\hline & SSD-HBW & 95.1 & 46.94 & 24.16 & 1592.6 & 132.99 & 8.91 \\
\hline & SSD-HBO & 61.48 & 4.08 & 0.37 & 337.5 & 123.51 & 7.42 \\
\hline \multirow[t]{3}{*}{12} & SAD-FBW & 91.85 & 46.84 & 7.88 & 357 & 112.14 & 3.32 \\
\hline & SSD-HBW & 90.52 & 15.32 & 0.88 & 674.5 & 114.59 & 3.58 \\
\hline & SSD-HBO & 81.07 & 5.69 & 0.00 & 316.7 & 110.38 & 3.21 \\
\hline
\end{tabular}


in SAD-FBW compared to SSD-HBW but SSD-HBO has less MU compared to SAD-FBW technique (Table 2). The comparison between the three techniques is shown in Diagram 1.

\section{Discussion}

Breast radiotherapy is a simple form of radiotherapy technique but can result in suboptimal target coverage and high OAR doses if proper techniques of planning are not applied. In general, for every patient, there is an optimum plan that treats the breast tissue while sparing the organs at risk. However, the technique one uses could vary depending on patient geometry or the technology available in the radiotherapy center, including the available treatment planning systems,

Table 2. Comparison of the three techniques showing the p-values (Wilcox-Signed Rank test-SAD-FBW technique was considered as the reference technique).

\begin{tabular}{ccccccccc}
\hline \multirow{2}{*}{ Parameters } & \multicolumn{3}{c}{ SAD-FBW } & \multicolumn{3}{c}{ SSD-HBW } & \multicolumn{3}{c}{ SSD-HBO } \\
\cline { 2 - 9 } & Mean & SD & Mean & SD & p-value & Mean & SD & p-value \\
\hline PTV Coverage (\%) & 94.78 & 2.27 & 92.33 & 4.69 & 0.005 & 75.058 & 11.9 & 0.002 \\
V107 (cc) & 81.65 & 37.49 & 49.72 & 22.75 & 0.012 & 35.64 & 15.13 & 0.077 \\
V110 (cc) & 23.64 & 15.93 & 10.85 & 6.62 & 0.084 & 10.10 & 5.86 & 0.266 \\
Monitor Unit & 709.43 & 320.41 & 1113.53 & 378.92 & 0.002 & 340.45 & 12.38 & 0.005 \\
Left Lung V20 (cc) & 108.42 & 43.64 & 109.61 & 43.84 & 0.109 & 104.21 & 43.29 & 0.004 \\
Mean Heart Dose (Gy) & 3.59 & 2.21 & 3.75 & 2.27 & 0.017 & 3.27 & 1.94 & 0.004 \\
\hline
\end{tabular}
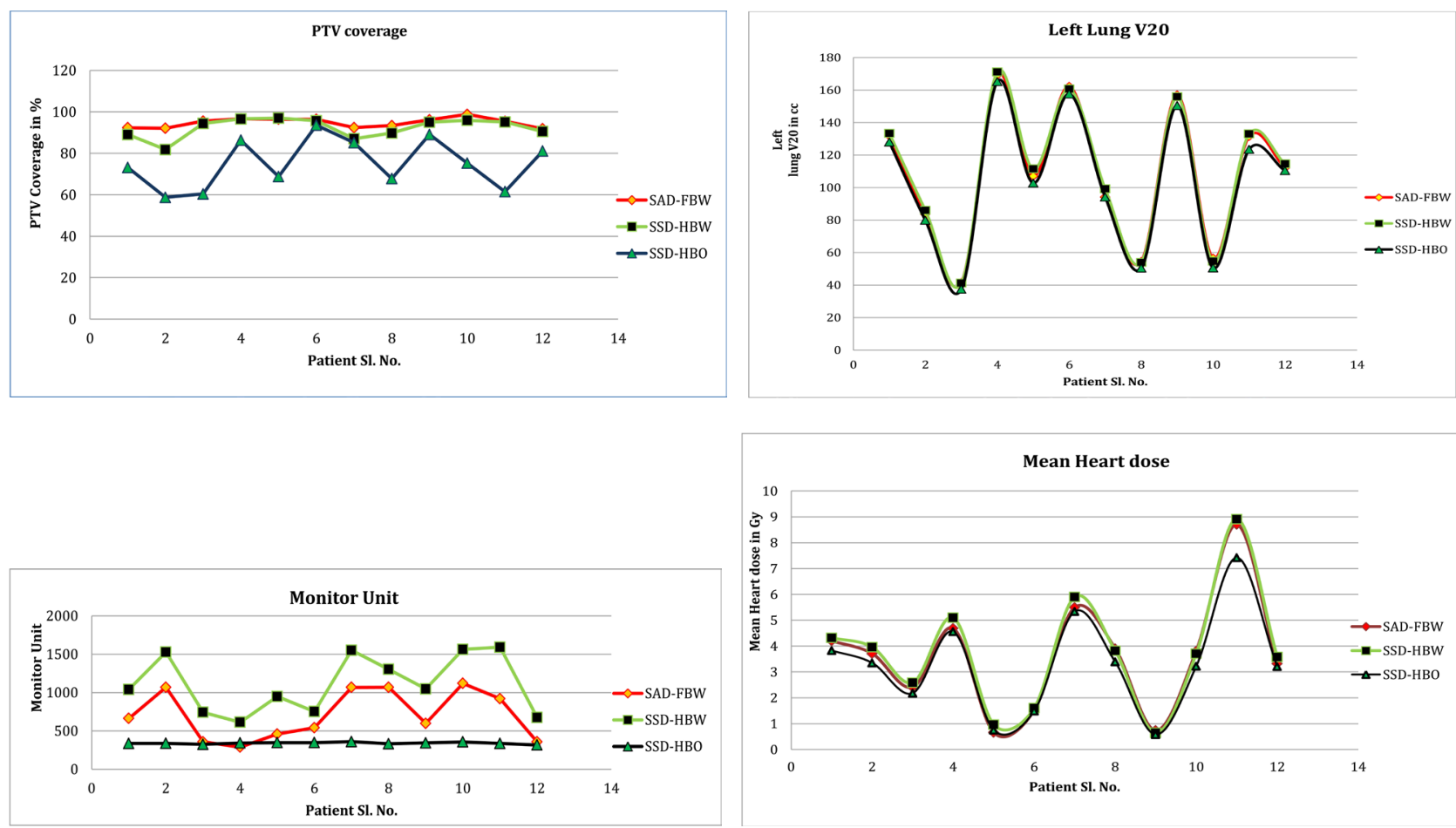

Diagram 1. Scatter diagram for PTV coverage, left lung V20, monitor unit and mean heart dose for three different planning techniques. 
beam energy, TPS algorithm, and the skills of the planner [6]. Since breast and chest wall are curved structures achieving the good coverage with tangential open beam techniques is difficult. This was also shown in our study that the PTV coverage is best when SAD-FBW technique is used compared to use of half beam block technique (Table 1) which is still a common practice in many centers across India. Use of SSD half Beam Block with wedge (SSD-HBW) is still having poor PTV coverage in some patients but with higher MU compared to SAD-FBW technique. The SSD-HBO (or use of Breast cone only) technique has the worst PTV coverage and should not be practiced in modern radiotherapy era. We accepted the PTV coverage below 95\% for some patients due to undue high hot spots. In order to achieve target coverage beyond $95 \%$, the $110 \%$ high dose region increased proportionately. The hot spots were more in BCS patients (total 4) compared to chest wall (total 8) patients. There are reasons to accept the plans with less than $95 \%$ coverage and with hot spots more than the standard ICRU guidelines. The most important reason is the irregular shape of the breast and chest wall resulting difficult planning volumes. Also the PTV changes from mid plane to superior and inferior borders along with change in chest wall shape (Figure 3). This wide variation might lead to under dosing in some areas and hot spots at variable locations leading to compromise in accepting plans as per standard guidelines. The variation of PTV shape at three different levels (Midplane, Superior and Inferior border) is shown in Table 3. The other reason is target contouring. RTOG atlas suggests drawing contours up to the skin level [9] and this might be the reason to have poor PTV coverage due to skin sparing effect of photon beams. Although we excluded the skin from our final PTV still the coverage was less in some patients. ESTRO consensus [15] has recommended taking $5 \mathrm{~mm}$ margin from skin to avoid this problem. Use of Bolus to achieve adequate target coverage in chest wall cases and increase surface dose is well documented and used by many clinicians around the world. Some previous breast cancer radiotherapy techniques, especially by using $2 \mathrm{D}$ techniques, involved some unwanted normal tissues by irradiation, including the heart and

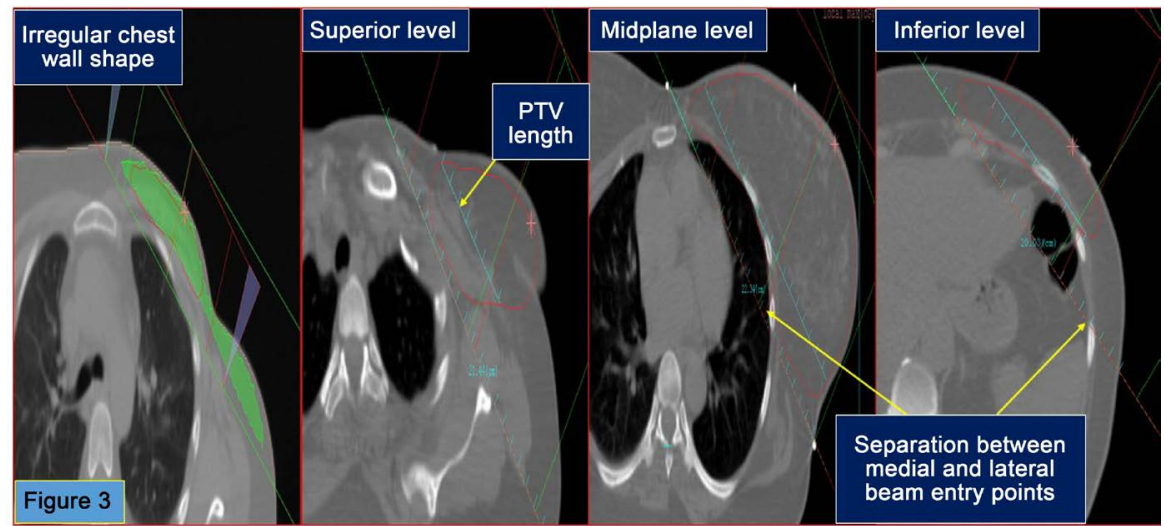

Figure 3. Irregular shape of chest wall leading to dose distribution in unwanted area with bi-tangential technique and variation in PTV distance at different levels without much variation in chest wall separation distance. WsW 
Table 3. PTV separation between medial and lateral edges at three different levels.

\begin{tabular}{cccc}
\hline \multirow{2}{*}{ Patient no. } & \multicolumn{2}{c}{ PTV separation between medial and lateral edge at three different levels $(\mathrm{cm})$} \\
\cline { 2 - 4 } 1 & Upper (Superior) & Mid Plane & Lower (Inferior) \\
\hline 2 & 9.23 & 12.83 & 10.91 \\
3 & 8.02 & 12.70 & 9.69 \\
4 & 8.40 & 16.52 & 7.02 \\
5 & 10.36 & 20.15 & 12.04 \\
6 & 14.65 & 19.30 & 12.27 \\
7 & 11.82 & 15.20 & 10.98 \\
8 & 11.10 & 13.58 & 13.31 \\
9 & 10.78 & 13.18 & 13.80 \\
10 & 8.94 & 14.18 & 12.31 \\
11 & 11.54 & 12.16 & 11.95 \\
12 & 13.64 & 14.21 & 12.46 \\
\hline
\end{tabular}

lungs [7]. Long term follow-up of the woman diagnosed with left-sided breast cancer has revealed an increased risk of death from heart disease, presumably because of some unwanted irradiation of cardiac structures using $2 \mathrm{D}$ technique [7] and in our study it is shown that in the standard SAD-FBW technique the mean heart doses are comparable to published data.

Nowadays many authors recommend the use of 3D planning with use of appropriate shielding and breath hold techniques to achieve best plan. Approval of an RT plan is an important part of the multidisciplinary treatment of breast cancer, thus the full picture of the patient should be considered in order to balance the compromises when evaluating and approving the RT plan based on the dose distribution.

The main objective of this dosimetric analysis was to show that use of SSD half beam blocks for the treatment of breast radiotherapy is obsolete and should not be considered. The use of SAD full beam techniques with optimal wedge application will give good coverage. The use of SAD technique will also help in easy setup and less treatment time (evident from the MU described in our study) compared to SSD techniques. Overall SAD techniques have several advantages over SSD techniques but mainly convenience, speed and accuracy, as with SSD techniques patients need to be realigned for the contralateral tangent which means a compromise on the three parameters listed. The longer a patient is on the table and unrestrained, with distractions, the more chances of movement and consequences. The use of SAD-FBW technique using conventional simulator without the availability of CT simulator is possible and is also done in many centers across India. But its actual procedure explanation is beyond the scope of this paper. 


\section{Conclusion}

SSD-HBW technique is found inferior to SAD-FBW technique. Comparatively greater total beam MU of SSD-HBW technique leads to more treatment time. SSD-HBO technique shows very poor PTV coverage. So, based on the results, one isocentric full beam with wedge technique provides significant advantages in terms of PTV coverage, normal tissue doses, easy set up and with minimum treatment time. We also recommend that centers treating patient with half beam SSD techniques should shift immediately to SAD techniques and if possible 3D planning for radiotherapy in breast patients.

\section{Acknowledgements}

I would like to sincerely thank Dr. Shashank Bansal and Mr. Manoj Kalita for their support, advice and help.

\section{References}

[1] WHO (2012) http://globocan.iarc.fr/

[2] Whelan, T.J. (2005) Use of Conventional Radiation Therapy as Part of Breast-Conserving Treatment. Journal of Clinical Oncology, 23, 1718-1725. https://doi.org/10.1200/JCO.2005.11.018

[3] Early Breast Cancer Trialists' Collaborative Group (EBCTCG) (2011) Effect of Radiotherapy after Breast-Conserving Surgery on 10-Year Recurrence and 15-Year Breast Cancer Death: Meta-Analysis of Individual Patient Data for 10801 Women in 17 Randomised Trials. Lancet, 378, 1707-1716. https://doi.org/10.1016/S0140-6736(11)61629-2

[4] Early Breast Cancer Trialists' Collaborative Group (EBCTCG) (2014) Effect of Radiotherapy after Mastectomy and Axillary Surgery on 10-Year Recurrence and 20-Year Breast Cancer Mortality: Meta-Analysis of Individual Patient Data for 8135 Women in 22 Randomised Trials. Lancet, 383, 2127-2135. https://doi.org/10.1016/S0140-6736(14)60488-8

[5] Morganti, A.G., et al. (2011) Forward Planned Intensity Modulated Radiotherapy (IMRT) for Whole Breast Postoperative Radiotherapy. Is It Useful? When? Journal of Applied Clinical Medical Physics, 12, 2. https://doi.org/10.1120/jacmp.v12i2.3451

[6] Haciislamoglu, E., et al. (2016) The Choice of Multi-Beam IMRT for Whole Breast Radiotherapy in Early-Stage Right Breast Cancer. Springer Plus, 5, 688-701. https://doi.org/10.1186/s40064-016-2314-2

[7] van der Laan, H.P., et al. (2008) Dosimetric Consequences of the Shift towards Computed Tomography Guided Target Definition and Planning for Breast Conserving Radiotherapy. Radiation Oncology, 3, 34-49. https://doi.org/10.1186/1748-717X-3-6

[8] van der Laan, H.P. (2010) Optimising CT Guided Radiotherapy for Breast Cancer. Doctoral Thesis, University of Groningen, Groningen, 171 p.

[9] RTOG (2017) https://www.rtog.org/CoreLab/ContouringAtlases/BreastCancerAtlas.aspx

[10] Mary Feng, M.D., Moran, J.M., Koelling, T., Chughtai, A., et al. (2009) Development and Validation of a Heart Atlas to Study Cardiac Exposure to Radiation Following Treatment for Breast Cancer. International Journal of Radiation Oncology Biology Physics, 79, 10-18. https://doi.org/10.1016/j.ijrobp.2009.10.058 
[11] Barrett, J.D.A. and Ash, D. (1992) Practical Radiotherapy Treatment Planning. 2nd Edition, CRC Press, Boca Raton.

[12] Rudat, V., et al. (2011) Tangential Beam IMRT versus Tangential Beam 3D-CRT of the Chest Wall in Post Mastectomy Breast Cancer Patients: A Dosimetric Comparison. Radiation Oncology, 6, 26-33. https://doi.org/10.1186/1748-717X-6-26

[13] Bencomo, J.A., et al. (2004) Anthropomorphic Breast Phantoms for Quality Assurance and Dose Verification. Journal of Applied Clinical Medical Physics, 5, 1-13. https://doi.org/10.1120/jacmp.v5i1.1992

[14] Urbańczyk, H., et al. (2005) The “One-Isocenter-Quarter-Beam” Technique as a Radiotherapy of Breast Cancer Patients. Reports of Practical Oncology and Radiotherapy, 337, 307-312. https://doi.org/10.1016/S1507-1367(05)71104-4

[15] Offersen, B.V., et al. (2015) ESTRO Consensus Guideline on Target Volume Delineation for Elective Radiation Therapy of Early Stage Breast Cancer. Radiotherapy and Oncology, 114, 3-10. https://doi.org/10.1016/j.radonc.2014.11.030 